\title{
Parameters Influencing the Dynamic Behaviour of the Carrying Structure of a Type H Portal Crane
}

\author{
Rade Vasiljević1,* - Milomir Gašić2 - Mile Savković2 \\ ${ }^{1}$ High Technical School of Vocational Studies, Serbia \\ 2 University of Kragujevac, Faculty of Mechanical and Civil Engineering, Serbia
}

\begin{abstract}
This paper deals with the problems investigating of parameters that influence the dynamic behaviour of the carrying structure of type $\mathrm{H}$ portal cranes with high carrying capacities excited by the motion of the crane and the load swing. The introduction of the paper presents the importance of the problem of the dynamics of structures. In accordance with the given problem, dynamic spatial models of the boom and portal of the crane were formed and solved. The main part of the paper presents a modal analysis and determines dynamic responses of the space structure with the application of the finite element method and direct integration. The influence of changes in speed and acceleration on the dynamic response of the structure was analysed. The influence of the change of boom rotation angle on the dynamic response of the structure was also analysed. The investigation results provide significant bases and conclusions for designing portal cranes and continuation of research in this field.
\end{abstract}

Keywords: type $\mathrm{H}$ portal crane, rotating boom, excitation, analysis of dynamic behaviour, finite element method, dynamic response

Highlights
- Definition of excitation in a portal crane.
- $\quad$ Dynamic model of portal crane boom.
- $\quad$ Dynamic response of the carrying structure to excitation.

\section{O INTRODUCTION}

Research into the dynamic behaviour of structures is present in mechanical and civil engineering. Structures of various purposes are exposed to variable influences. These influences can be common [1], which are variable only in time, and influences of moving loads, which are variable in time and space [2] and [3]. The action of these influences on structures is reflected in the occurrence of dynamic displacements, velocities, and accelerations of the structure.

In mechanical engineering, this problem is present, above all, in the field of crane engineering. One of the representatives of crane machines, which are the subject of this paper, is the portal-rotating crane. These are crane machines with a boom that is connected to the portal and that has the possibility of a $360^{\circ}$ rotation. These cranes are interesting for dynamic analysis due to their high carrying capacities and the fact that they have a small base in proportion to their height. In portal-rotating cranes, there are influences that are variable in time. Load swing contributes significantly to the dynamic loading of cranes. The importance of the problem of load swing is considered and confirmed in papers [1] and [4]. Furthermore, the importance of this problem is defined in standards [5].

The first papers considered planar dynamic models, and later papers published specifically in the field of dynamics of portal cranes mostly treated planar dynamic models [6] and [7]. The authors of this paper found a small number of papers with dynamic spatial models which dealt with the problems of the dynamic behaviour of portal-rotating cranes [8]. Therefore, the search was directed, in a wider sense, to the group of rotating cranes. First, a group of dynamic models that deal with the problems of the dynamic behaviour of rotating cranes was considered [9] to [11]. Next, a group of dynamic models that deal with the problems of the control of rotating cranes was reviewed [12] and [13].

In almost all the above-mentioned papers [6] to [13], the authors modelled portal-rotating cranes as direct or discrete-continuous models. Vasiljević et al. show [14] that more accurate results are obtained by the application of consistent masses.

The papers mostly provide an investigation of the dynamic models of only booms of portal cranes, but few of them investigate dynamic models which take into account the carrying structure.

In accordance with this fact, this paper should contribute to the knowledge of the dynamic behaviour of the carrying structure of the portal crane excited by the motion of the crane and the load swing. The solution to this problem requires appropriate modelling of the boom and the carrying structure. The approach to modelling of the dynamic model of 
the portal crane is such that the whole portal crane is divided into two subsystems, i.e. the carrying structure and the boom. The relationship between the carrying structure and the boom is simplified by reducing the influence of load and the dead weight of the boom to the corresponding points of the portal. The carrying structure of the portal crane is modelled as a spatial linear beam. In reality, spatial linear beams are systems with continuously distributed masses, whereas the spatial model of the carrying structure is more complex. For these reasons, the continuously distributed mass is replaced with the consistent mass. Hence, the consistent mass matrix of the system is formulated on the basis of the same interpolation functions that are used for the derivation of stiffness matrices. The spatial linear beam is modelled by means of a space girder element, with two node points and six unknowns in each node.

For the adopted dynamic model of the portalrotating crane, modal analysis of oscillation was conducted in the first step, and it forms the basis for a good understanding of the dynamic characteristics of the carrying structure. In the second step, the dynamic response of the portal-rotating crane to excitation was determined.

\section{PROBLEM SETTING}

Oscillation results from the elasticity of mechanisms and the carrying structure due to the action of inertial forces. The shorter the time of action of those forces, the more unfavourable the oscillations.

The first main problem in the investigation of the dynamic behaviour of portal cranes is the definition of excitation. The structure, i.e. the base of the crane considered, can perform only rectilinear motion from point to point. The kinematic values of the motion of the portal crane are the speed of motion, acceleration and the distance passed. The trapeze velocity profile is most frequently used for the calculation of mechanisms for the motion of cranes. Accordingly, in this paper, it is assumed that the crane moves along its track with the trapeze velocity profile over the course of one cycle (Fig. 1). From Fig. 1, it is seen that the motion of the structure consists of three phases. In phase 0 to 1 (time $t_{a}$ ), the structure accelerates with the constant acceleration $a_{a}$ so that the velocity linearly increases, and at the end of the first phase, it reaches the velocity $v_{u}$. The second phase, 1 to 2 , is the phase of uniform motion of the structure during the time $t_{u}$, which is characterized by the constant velocity $v_{u}$. In phases 2 to 3 (time $t_{d}$ ), the structure constantly decelerates with the deceleration $a_{d}$ until it stops. The distance passed by the structure is determined based on the profile of velocities (Fig. 1). The presented profile of velocities will be used for obtaining the dynamic response of the carrying structure excited by the motion of the crane and the load swing.

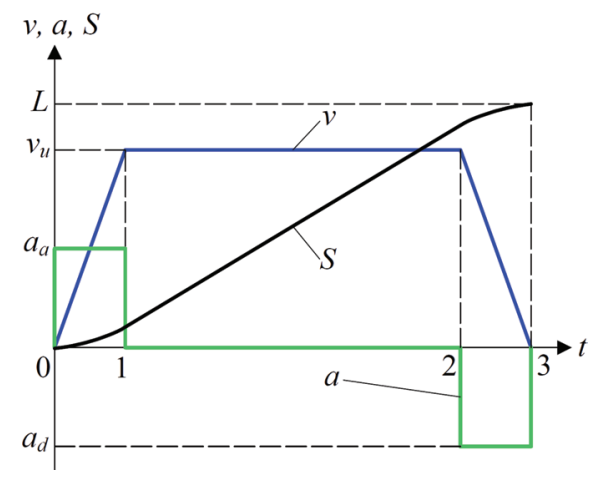

Fig. 1. Kinematic diagrams of motion of the crane

The second main problem in the investigation of the dynamic behaviour of portal-rotating cranes is the adoption of the appropriate model. Formation of the portal crane model is approached in such a way that the influence of loads and the boom is reduced to the corresponding points of the carrying structure of the crane. An analogous approach was applied in the investigation of the dynamic behaviour of the reloading bridge. It is shown in [15].

\section{DYNAMIC MODEL OF THE BOOM}

\subsection{Model Description}

The boom is a constituent part of the portal-rotating crane. The subsystem of the boom consists of the carrying structure and the rope system. The carrying structure of the boom is connected to the platform by joints.

In the system of the portal crane, the boom is observed, by idealization, as an independent subsystem. Accordingly, Fig. 2 presents a dynamic equivalent model of the portal crane boom.

The model was formed for the needs of determination of dynamic loads. The boom is observed as an independent subsystem which oscillates. A dynamic equivalent model is formed in such a way that it both keeps the main dynamic characteristics of the boom and that the defined problem could be mathematically solved.

From Fig. 2 it is seen that the subsystem of the boom with a load is represented with two lumped masses, two lightweight bars, and a circular disc. 
Discretization of the carrying structure of the boom was performed on a lightweight bar with a reduced mass on the tip. In other words, the carrying structure of the boom is represented by a lightweight bar with the length $L_{b}$ and the mass $m_{1}$, which is reduced to the tip of the boom. The rope system of the boom is represented by a non-elastic lightweight bar with the length $L_{r}$ and the mass $m_{2}$, which allows load swing. It means that the tip of the boom, i.e. the mass $m_{1}$ and the mass $m_{2}$, are joined by the non-elastic lightweight bar with the length $L_{r}$. The rotating column is represented by a circular disc with the axial moment of inertia $J$ and the moment of rotation $T$. The distance between the boom joint and the column axis is $r$.

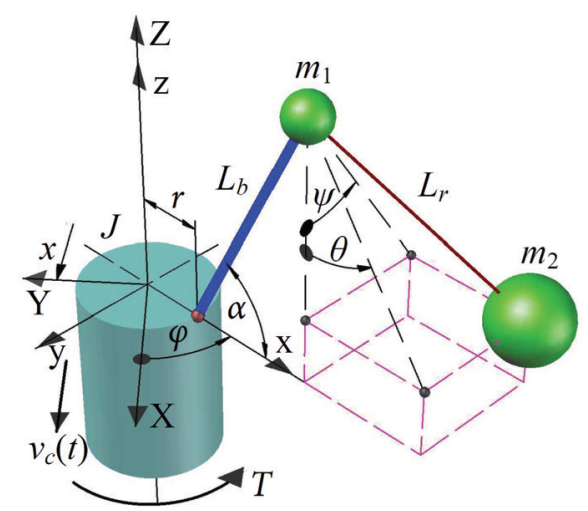

Fig. 2. Dynamic model of the boom

Based on the recommendations in [15], the reduced mass $m_{1}$ can be determined according to the following relation:

$$
m_{1}=\left(\frac{1}{4} \ldots \frac{1}{3}\right) m_{b},
$$

where $m_{b}$ is the boom mass.

\subsection{Mathematical Formulation}

The Lagrange equations of the second kind will be used for the setting of the mathematical formulation of the formed dynamic model of the boom. The equations of motion of the boom elements are set based on the dynamic equivalent model presented in Fig. 2. read:

The dynamic equations of motion of the system

$$
\begin{aligned}
\ddot{\theta}(t)+\omega_{l}^{2} \sin \theta(t) & =-\frac{1}{L_{r}} \ddot{x}(t) \cos \varphi, \\
\ddot{\psi}(t)+\omega^{2} \sin \psi(t) & =-\frac{1}{L_{r}} \ddot{x}(t) \sin \varphi, \\
J \ddot{\varphi}(t) & =T,
\end{aligned}
$$

where $\theta$ is the angle of oscillation of load in the longitudinal direction, $\psi$ is the angle of oscillation of load in the side (lateral) direction, $\varphi$ is the angle of rotation of the column, i.e. the boom, $x$ is the rectilinear motion of the boom, and $\omega$ is the circular frequency of load oscillation.

\subsubsection{Load Oscillation}

The laws of non-attenuated oscillations of the load as a function of time along the generalized coordinates $\theta$ and $\psi$ due to the crane acceleration given by the diagram according to Fig. 1 will be determined from Eqs. (2a) and (2b).

In the first step, the motion of load, i.e. mass $m_{2}$ in the longitudinal direction along the generalized coordinate $\theta$ is observed in such a way that it is taken out of the system, Fig. 3.

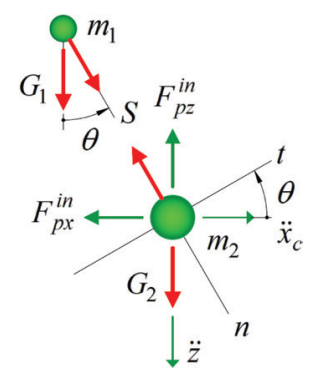

Fig. 3. Load oscillation in the longitudinal direction

For determination of the law of load swing, the differential equation of relative motion of the particle, i.e. mass $m_{2}$, is defined:

$$
m_{2} \vec{a}_{r}=\vec{F}_{p x}^{i n}+\vec{F}_{p z}^{i n}+\vec{G}_{2}+\vec{S}
$$

Eq. (3) is projected onto the axes $t$ and $n$ of the natural trihedron, which is connected to the mass $m_{2}$. The details of obtaining the law of load oscillation in the longitudinal direction are given. As there is an analogy between load oscillation in these two directions, only the final form will be given for the side direction.

The law of load oscillation in the longitudinal direction obtains the form:

$$
\ddot{\theta}(t)+\frac{g}{L_{r}} \sin \theta(t)=-\frac{1}{L_{r}} \ddot{x}(t) \cos \theta(t) \cos \varphi .
$$

In Eq. (4), the relation $g / L_{r}$ represents the quadrant circular frequency of the load $\omega^{2}$. For the case of small oscillations, the approximation that the angle $\theta$ is small can be introduced, so that $\sin \theta \approx \theta$ and $\cos \theta \approx 1$. Furthermore, the replacement $a=\ddot{x}(t)$ is 
introduced. Eq. (4) is linearized so that it obtains the following form:

$$
\ddot{\theta}(t)+\omega^{2} \theta(t)=-\frac{1}{L_{r}} a(t) \cos \varphi .
$$

Eq. (5) is most suitably solved by the method of Laplace transformation. In the first step, it is determined that:

$$
L\{(s)\}=-\frac{1}{L_{r}} L\left\{\frac{a(s)}{\left(s^{2}+\omega^{2}\right)}\right\} \cos \varphi .
$$

From the kinematic diagrams from Fig. 1, the acceleration diagram is particularly suitable for solving Eq. (6). This diagram is presented in Fig. 4. It is adopted that $a_{a}=a_{d}=h$ and $t_{a}=t_{d}=\tau$. For previous calculations, it can be adopted that $0.5\left(t_{a}+t_{d}\right)=3$ to $5 \mathrm{~s}$.

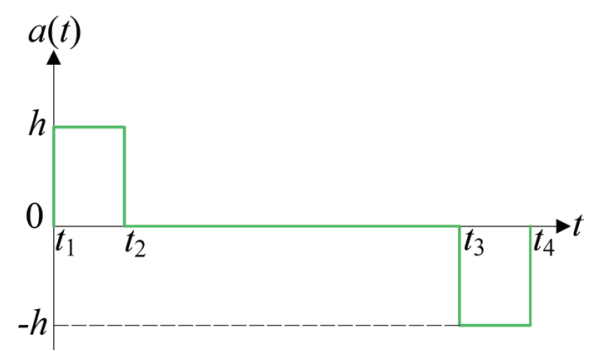

Fig. 4. Acceleration diagram

Now, according to the diagram of change of acceleration in time, Fig. 4, the expression for acceleration in the Laplace domain can be represented in the following form:

$$
a(s)=\frac{h}{s}\left(1-e^{t_{2} s}-e^{t_{3} s}+e^{t_{4} s}\right),
$$

where $h$ is the amplitude of input acceleration and $t_{i}$ is the corresponding time step in the acceleration diagram.

Eq. (6) obtains the form:

$$
L\{\theta(s)\}=-\frac{h}{L_{r}} L\left\{\frac{1-e^{t_{2} s}-e^{t_{3} s}+e^{t_{4} s}}{s\left(s^{2}+\omega^{2}\right)}\right\} \cos \varphi .
$$

Finally, the law of load oscillation in the longitudinal direction is obtained by transforming Eq. (8) for $\theta(s)$ in the time domain by using the inverse Laplace transform.

$$
\begin{aligned}
& \theta(t)=-\frac{h}{L_{r} \omega^{2}} \cos \varphi\{(1-\cos \omega t)- \\
& {\left[1-\cos \omega\left(t-t_{2}\right)\right] \text { HeavisideTheta }\left(t-t_{2}\right)-} \\
& {\left[1-\cos \omega\left(t-t_{3}\right)\right] \text { HeavisideTheta }\left(t-t_{3}\right)+} \\
& \left.\left[1-\cos \omega\left(t-t_{4}\right)\right] \text { HeavisideTheta }\left(t-t_{4}\right)\right\}, \\
& \psi(t)=-\frac{h}{L_{u} \omega^{2}} \sin \varphi\{(1-\cos \omega t)- \\
& {\left[1-\cos \omega\left(t-t_{2}\right)\right] \text { HeavisideTheta }\left(t-t_{2}\right)-} \\
& {\left[1-\cos \omega\left(t-t_{3}\right)\right] \text { HeavisideTheta }\left(t-t_{3}\right)+} \\
& \left.\left[1-\cos \omega\left(t-t_{4}\right)\right] \text { HeavisideTheta }\left(t-t_{4}\right)\right\} .
\end{aligned}
$$

The obtained laws of load oscillation (Eqs. (9) and (10)) represent Heaviside (step) functions with a linear polynomial.

The load swing contributes the most to the instability and overturning of the crane when the angle of load oscillation reaches its highest point. This case occurs when the impulses of time calculation are mutually in phase. In that case, load oscillation caused by every step of acceleration is constructively added and produces the highest amplitude of load oscillation.

Eqs. (9) and (10) show that the maximum angle of oscillation appears when the following conditions are fulfilled:

- each of the cosine members within Eqs. (9) and (10) is in phase,

- multiplying the cosine members within Eqs. (9) and (10) by the corresponding step of the function HeavisideTheta results in obtaining the value equal to 1 (it is necessary to have a sufficient time of operation, $t \geq t_{4}$, in order to achieve acceleration according to the diagram).

The cosine members within Eqs. (9) and (10) are in phase when four steps of the acceleration diagram are performed by their constructive adding in perfect time. In the worst case, the angle of oscillation increases four times in relation to the oscillation caused by one step of input acceleration. The maximum angle of load oscillation in the longitudinal and side directions is equal to:

$$
\left|\theta_{\max }\right|=\left|\psi_{\max }\right|=4 \frac{h}{g} .
$$

To produce maximum load swing, it is necessary to have acceleration steps completely in phase, i.e. it is necessary to fulfil two conditions.

The first condition requires that the time interval between the first and the second steps, as well as 
between the third and the fourth ones should be equal to one half of the oscillation period $\tau$ :

$$
t_{2}-t_{1}=t_{2}=(0.5+n) \tau \text {, and } t_{4}-t_{3}=(0.5+n) \tau,
$$

where $n=1,2,3, \ldots$

The time delay is necessary because the steps in sets have different designations. Therefore, the time delay is equivalent to the excitation of the phase delay $\pi$ according to the opposite signs of function, which excludes the possibility of the change of phases (and brings two functions in phase).

The second condition requires that the time interval between the second and the third impulses must contain the time delay of the multiple period $\tau$ :

$$
t_{3}-t_{2}=n \tau,
$$

where $n=1,2,3, \ldots$

In other words, this holds because the second and the third steps have the same sign so that the change of phase by $2 \pi$ requires that the second and the third steps should be in phase.

\subsubsection{Dynamic Loads of the Portal}

In accordance with the adopted generalized coordinates of oscillation of the dynamic model of the boom, the dynamic moment of bending occurs in two directions:

- in the longitudinal direction, and

- in the side (lateral) direction.

The dynamic moment of bending in the longitudinal direction reads, Fig. 2:

$$
\begin{aligned}
& M_{d y n, l}=m_{1} g\left(L_{b} \cos \alpha+r\right)+ \\
& m_{2}\left(g+L_{r} \dot{\theta}^{2} \cos \theta\right)\left(L_{r} \sin \theta+L_{b} \cos \alpha+r\right) \\
& +m_{2} L_{r} \dot{\theta}^{2} \sin \theta\left(L_{b} \sin \alpha-L_{r} \cos \theta\right)+ \\
& m_{2} L_{r} \dot{\psi}^{2} \cos \psi\left(L_{r} \cos \alpha+r\right) .
\end{aligned}
$$

The dynamic moment of bending in the side direction reads, Fig. 2:

$$
\begin{aligned}
& M_{d y n, s}=m_{2}\left(g+L_{r} \dot{\psi}^{2} \cos \psi\right) L_{r} \sin \psi+ \\
& m_{2} L_{r} \dot{\psi}^{2} \sin \psi\left(L_{b} \sin \alpha-L_{r} \cos \psi\right) .
\end{aligned}
$$

\section{FINITE ELEMENT MODEL OF THE STRUCTURE}

\subsection{Model Description}

The whole portal-rotating crane is divided into two subsystems: the moving structure, and the boom. The relationship between the structure and the boom is simplified in such a way that the influence of load and the dead weight of the boom is reduced to the points of the upper and lower supports of the boom.

The type $\mathrm{H}$ carrying structure of the considered portal crane is shown in Fig. 5.

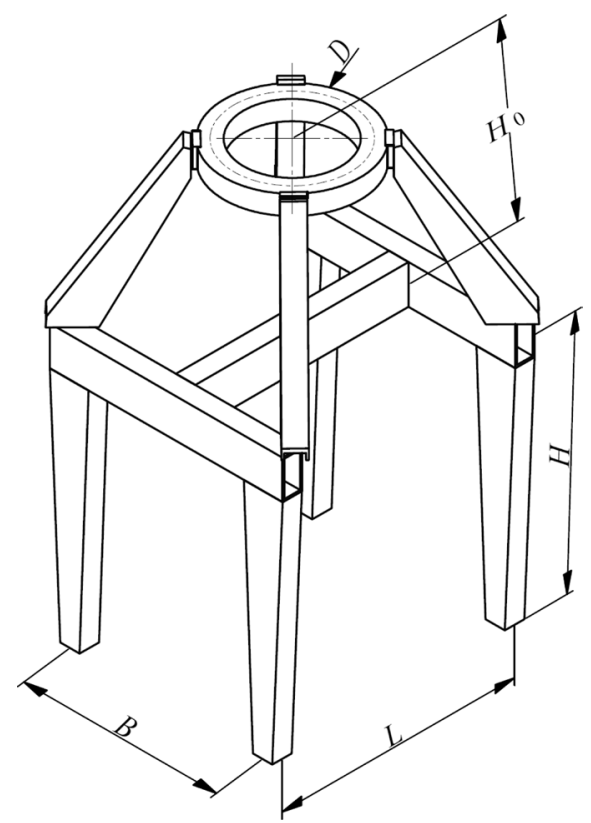

Fig. 5. Carrying structure (portal) of type " $\mathrm{H}^{\prime \prime}$

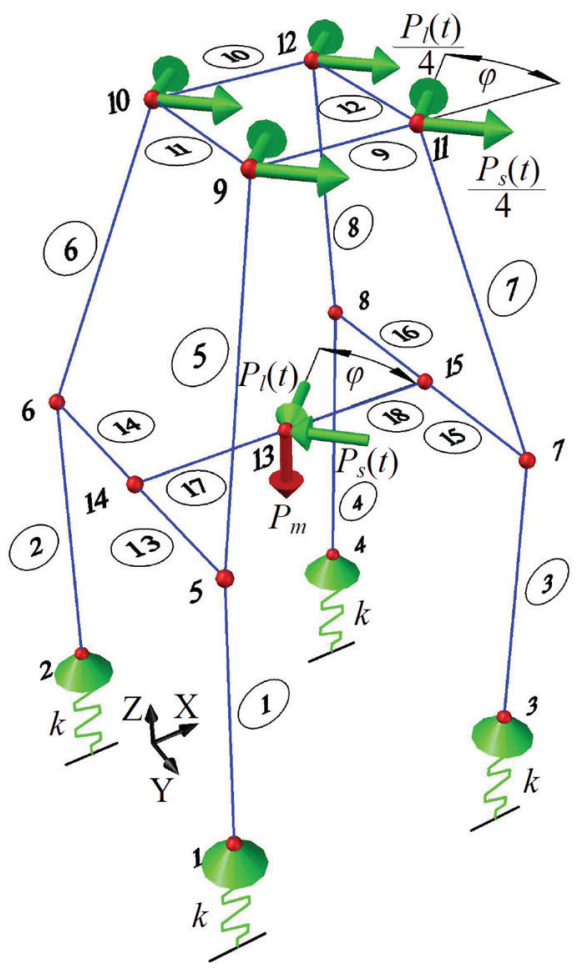

Fig. 6. Finite element model of the type $H$ structure 
The carrying structure is a rigid spatial frame. Its base has dimensions $L \times B$. The main structural parts of the carrying structure are the legs, the slanted columns, and the lower and the upper beams. The legs are identical (height $H$ ) and stand at the same level. The slanted columns are identical (length $C$ ) and they are connected to the legs as well as to the upper and lower beams. The distance between the upper and the lower beams is equal to $H_{0}$. The upper beam has the function of the upper support of the boom, and it is made of a circular ring with the diameter $D$. The lower beam has the function of the lower support of the boom, and it is made of a type $\mathrm{H}$ frame.

The finite element model of the structure of the considered portal crane is shown in Fig. 6.

Taking into account that the boom rotates around its axis, it can be concluded that the assumed planar model is not sufficient for describing the dynamic behaviour of the considered portal-rotating crane. The spatial model of the portal crane, to which dynamic load is reduced, is formed in accordance with the spatial dynamic model of the boom.

Discretization of the carrying structure of the crane was performed on 18 finite beam elements connected by nodes. The whole system has 82 degrees of freedom of motion. The legs were modelled as one finite element with the characteristics $A_{1}, I_{x 1}$, $I_{y 1}, I_{z 1}$. The slanted columns were modelled as one finite element with the characteristics $A_{5}, I_{x 5}, I_{y 5}, I_{z 5}$. The upper beam (the circular ring with the diameter $D$ ) was divided into four finite elements with the characteristics $A_{9}, I_{x 9}, I_{y 9}, I_{z 9}$. The lower beam (the type $\mathrm{H}$ frame) was divided into six finite elements with the characteristics $A_{13}, I_{x 13}, I_{y 13}, I_{z 13}$. The supporting structure rests on four elastic supports with the stiffness $k$.

The main mechanical characteristics of the carrying structure, i.e. of all finite elements of the formed spatial model are the module of elasticity $E$, the slipping module $G$, and the density $\rho$.

The dynamic moments, Eqs. (14) and (15), are reduced to the combination of horizontal dynamic forces $P_{l}(t)$ and $P_{s}(t)$ in the nodes of finite elements of the upper beam and the central node of the lower beam. The position of these forces relative to the longitudinal direction (the axis $\mathrm{X}$ ) is defined by the angle $\varphi$, which represents the angle of boom rotation.

The dynamic forces $P_{l}(t)$ and $4 \times P_{l}(t) / 4$ make a combination of forces that opposes the dynamic moment of overturning in the longitudinal direction $M_{d y n, l}$ of the platform, i.e. the rotating part of the crane:

$$
M_{d y n, l}=P_{l}(t) \cdot H_{0} .
$$

The dynamic forces $P_{s}(t)$ and $4 \times P_{s}(t) / 4$ make a combination of forces which opposes the dynamic moment of overturning in the side direction $M_{d y n, s}$ of the platform:

$$
M_{d y n, s}=P_{s}(t) \cdot H_{0} .
$$

The vertical static force $P_{m 1}$ of the load mass $m_{1}$ is reduced to the central node of the lower beam (the type $\mathrm{H}$ frame). The vertical static force $P_{m 2}$ of the reduced boom mass $m_{2}$ is also reduced to the central node of the lower beam. The total vertical static force $P_{m}$ that acts in the central node of the lower support of the boom is equal to the sum of the force of the load mass and the force of the reduced boom mass:

$$
P_{m}=P_{m_{1}}+P_{m_{2}} \text {. }
$$

The characteristic nodes of the structure model for obtaining dynamic response of the structure to excitation are the nodes at the point of elastic supports (points of wheel-rail contact).

\subsection{Mathematical Formulation}

In accordance with the formed mathematical model of the portal crane, the differential equation of dynamic equilibrium, i.e. forced oscillations of the carrying structure reads:

$$
[\mathbf{M}]\{\ddot{\mathbf{U}}\}+[\mathbf{K}]\{\mathbf{U}\}=\{\mathbf{P}(\mathbf{t})\},
$$

where $[\mathbf{M}]$ is the mass matrix of the system, $[\mathbf{K}]$ is the stiffness matrix of the system, $\{\ddot{\mathbf{U}}\}$ the vector of acceleration of generalized coordinates of the carrying structure, $\{\mathbf{U}\}$ the vector of displacement of generalized coordinates of the structure, and $\{\mathbf{P}(\mathbf{t})\}$ the vector of external load of the structure nodes.

Eq. (19) is used for studying the dynamic response of the structure due to the motion of the crane. It can be decomposed to the equilibrium conditions of active and inertial forces in the direction of unknown and known displacements so that it gains the form:

$$
\left[\begin{array}{ll}
\mathbf{M}_{\mathrm{uu}} & \mathbf{M}_{\mathrm{uk}} \\
\mathbf{M}_{\mathrm{ku}} & \mathbf{M}_{\mathrm{kk}}
\end{array}\right]\left\{\begin{array}{c}
\ddot{\mathbf{U}}_{\mathrm{u}} \\
\ddot{\mathbf{U}}_{\mathrm{k}}
\end{array}\right\}+\left[\begin{array}{ll}
\mathbf{K}_{\mathrm{uu}} & \mathbf{K}_{\mathrm{uk}} \\
\mathbf{K}_{\mathrm{ku}} & \mathbf{K}_{\mathrm{kk}}
\end{array}\right]\left\{\begin{array}{c}
\mathbf{U}_{\mathbf{u}} \\
\mathbf{U}_{\mathbf{k}}
\end{array}\right\}=\left\{\begin{array}{l}
\mathbf{P}_{\mathbf{u}}(\mathbf{t}) \\
\mathbf{P}_{\mathbf{k}}(\mathbf{t})
\end{array}\right\} \text {. }
$$

As the displacements and accelerations of the supports in horizontal directions are equal to zero $\left(\mathbf{U}_{\mathbf{k}}=0\right.$ and $\left.\ddot{\mathbf{U}}_{\mathbf{k}}=0\right)$, Eq. (20) obtains the form:

$$
\left[\mathbf{M}_{\mathbf{u u}}\right]\left\{\ddot{\mathbf{U}}_{\mathbf{u}}\right\}+\left[\mathbf{K}_{\mathbf{u u}}\right]\left\{\mathbf{U}_{\mathbf{u}}\right\}=\left\{\mathbf{P}_{\mathbf{u}}(\mathbf{t})\right\} .
$$

Natural frequencies of the structure are obtained by solving the algebraic equation:

$$
\operatorname{det}\left(\mathbf{K}_{\mathbf{u u}}-\omega_{s t}^{2} \mathbf{M}_{\mathbf{u u}}\right)=0 .
$$


In the direction of unknown displacements, the mass submatrix of the whole system is equal to the matrix of the carrying structure of the portal crane:

$$
\mathbf{M}_{\mathbf{u u}}=\left[\mathbf{M}_{\mathbf{u u}}\right]_{82 \times 82} \text {. }
$$

In the direction of unknown displacements, the stiffness submatrix of the whole system includes the matrix of the structure and the stiffness matrix of elastic supports:

$$
\mathbf{K}_{\mathrm{uu}}=\left[\mathbf{K}_{\mathrm{uu}}\right]_{82 \times 82}+[\mathbf{k}]_{16 \times 16} .
$$

In the direction of unknown displacements, the subvector of external dynamic forces of the whole system is equal to the subvector of external dynamic forces of the carrying structure of the portal crane:

$$
\mathbf{P}_{\mathbf{u}}(\mathbf{t})=\left\{\mathbf{P}_{\mathbf{u}}(\mathbf{t})\right\}_{82 \times 1} .
$$

The subvector of external dynamic forces $\mathbf{P}_{\mathbf{u}}(\mathbf{t})$ can be expressed in the following form:

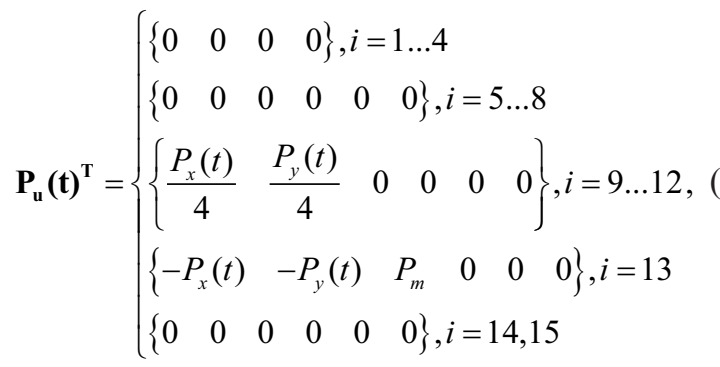

where:

$$
\begin{aligned}
& P_{x}(t)=P_{l}(t) \cos \varphi+P_{s}(t) \sin \varphi, \text { and } \\
& P_{y}(t)=-P_{l}(t) \sin \varphi+P_{s}(t) \cos \varphi .
\end{aligned}
$$

\section{NUMERICAL RESULTS AND DISCUSSION}

For determination of numerical values of parameters influencing the dynamic behaviour of the carrying structure of the type $\mathrm{H}$ portal crane and the formation of appropriate conclusions, the initial data were defined based on the solutions derived and manufacturers' catalogues: $v_{c}=0.6 \mathrm{~m} / \mathrm{s} ; m_{t}=10000 \mathrm{~kg} ; m_{s}=9200 \mathrm{~kg}$; $r=1 \mathrm{~m} ; L_{b}=30 \mathrm{~m} ; H=6 \mathrm{~m} ; H_{0}=3.675 \mathrm{~m} ;$ $A_{n}=31500 \times 10^{-6} \mathrm{~m}^{2}, l_{n}=6 \mathrm{~m}, I_{y n}=3.117 \times 10^{-3} \mathrm{~m}^{4}$, $I_{z n}=1.391 \times 10^{-3} \mathrm{~m}^{4}, I_{x n}=2.776 \times 10^{-3} \mathrm{~m}^{4}(n=1$ to 4$)$; $A_{n}=31500 \times 10^{-6} \mathrm{~m}^{2}, l_{n}=4.243 \mathrm{~m}, I_{y n}=3.117 \times 10^{-3} \mathrm{~m}^{4}$, $I_{z n}=1.391 \times 10^{-3} \mathrm{~m}^{4}, I_{x n}=2.776 \times 10^{-3} \mathrm{~m}^{4}(n=5$ to 8$)$; $A_{n}=26500 \times 10^{-6} \mathrm{~m}^{2}, l_{n}=3 \mathrm{~m}, I_{y n}=1.303 \times 10^{-3} \mathrm{~m}^{4}$, $I_{z n}=1.066 \times 10^{-3} \mathrm{~m}^{4}, I_{x n}=1.630 \times 10^{-3} \mathrm{~m}^{4}(n=9$ to 12$)$; $A_{n}=25200 \times 10^{-6} \mathrm{~m}^{2}, l_{n}=3 \mathrm{~m}, I_{y n}=1.115 \times 10^{-3} \mathrm{~m}^{4}$, $I_{z n}=1.119 \times 10^{-3} \mathrm{~m}^{4}, I_{x n}=1.611 \times 10^{-3} \mathrm{~m}^{4}(n=13$ to 18$)$;
$E=2.1 \times 10^{11} \mathrm{~N}^{2} ; G=0.8 \times 10^{11} \mathrm{~N}^{2} \mathrm{~m}^{2} ; \rho=7850 \mathrm{~kg} / \mathrm{m}^{3} ;$ $k=10^{7} \mathrm{~N} / \mathrm{m} ; g=9.81 \mathrm{~m} / \mathrm{s}^{2}$.

\subsection{Excitation}

The motion of the crane along the tracks is described by the diagram of velocity $v_{c}(t)$, Fig. 7 .

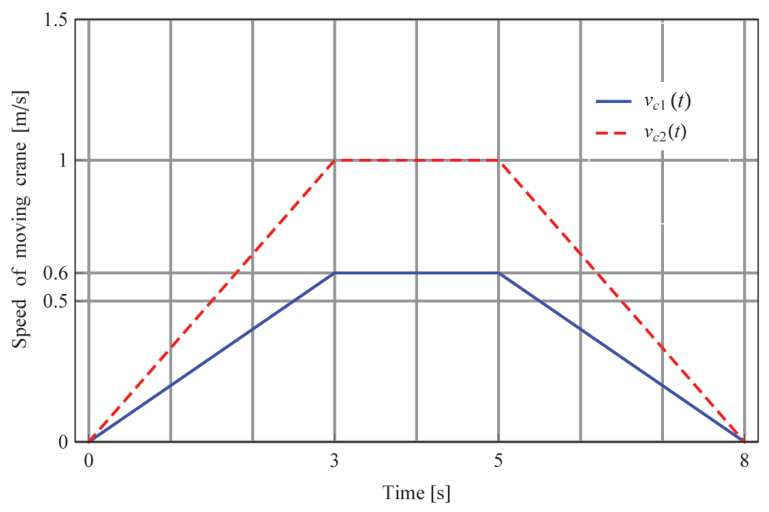

Fig. 7. Time diagram of the crane velocity $v_{c}(t)$

The change of angle of load oscillation in the longitudinal direction $\boldsymbol{\theta}$ according to Eq. (9) is presented in Fig. 8. The maximum angle of load oscillation is $0.0815 \mathrm{rad}$.

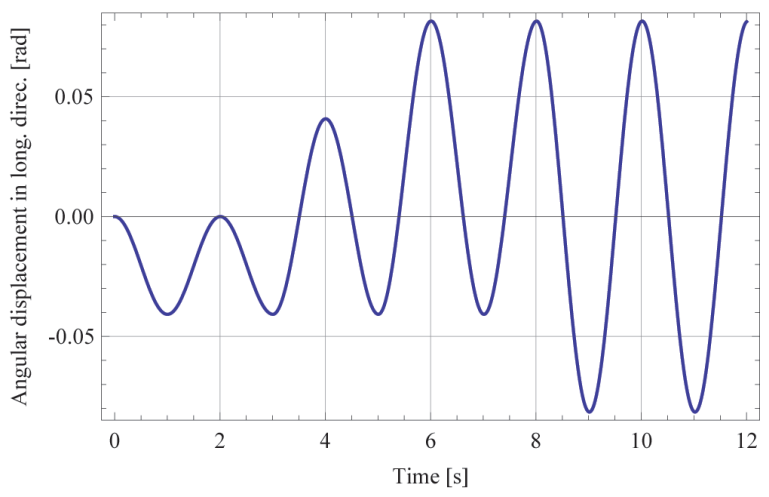

Fig. 8. Angle of load oscillation in the longitudinal direction

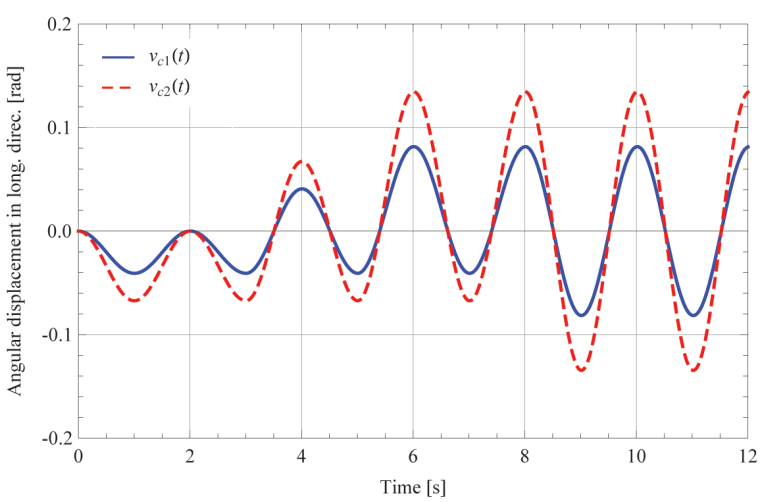

Fig. 9. Angle of load oscillation in the longitudinal direction for different speeds of motion of the crane 
Fig. 9, based on Eq. (9), presents the angle of load oscillation in the longitudinal direction for two different speeds of the crane.

The speed $v_{c 1}$ is the nominal speed of the crane, whereas the speed $v_{c 2}$ represents the extreme performance of the portal crane. Fig. 9 shows that the angle of load oscillation increases with the increase in the speed of the motion of the portal.

The change of the angle of load oscillation in the lateral direction $\psi$ is completely the same.

The change of the dynamic bending moment in the longitudinal and side directions $(\boldsymbol{\theta}, \boldsymbol{\psi})$ according to Eqs. (14) and (15) is presented in Figs. 10 and 11.

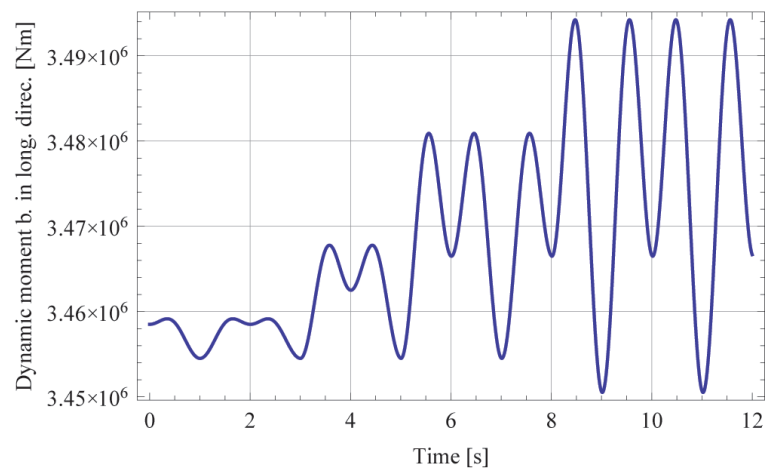

Fig. 10. Dynamic bending moment in the longitudinal direction

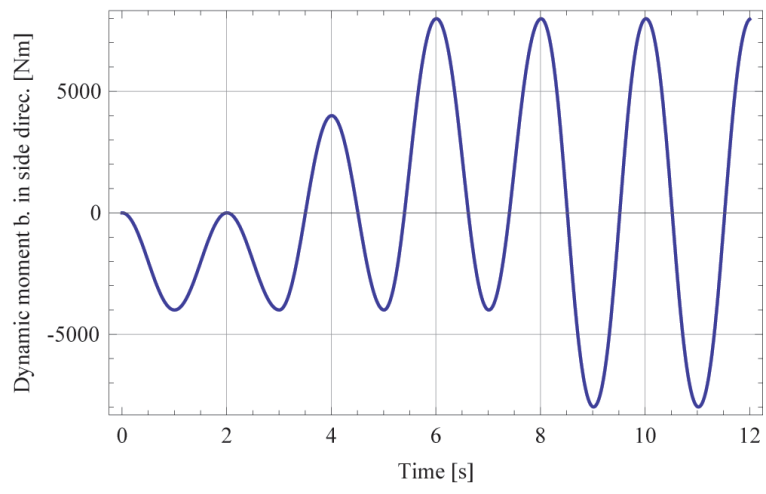

Fig. 11. Dynamic bending moment in the side direction

Detailed analysis of excitation in the portal crane is shown in [16].

\subsection{Response of the Carrying Structure to Excitation}

\subsubsection{Modal Analysis}

Table 1 presents the values of the first two frequencies of oscillation of the carrying structure of the portalrotating crane, Eq. (22).

The values of natural frequencies represent the first and most important element of estimation of dynamic stability of cranes in the first phase of the design of new solutions [14]. The crane has good dynamic behaviour if its first frequency of oscillation is high. According to this aspect, the carrying structure of the considered portal crane has favourable dynamic stability $\left(f_{1}=2.65 \mathrm{~Hz}\right)$.

Table 1. Frequencies

\begin{tabular}{cccc}
\hline Mode no. & Period $T_{\text {st }}[\mathrm{s}]$ & Circ. freq. $\omega_{\text {st }}[\mathrm{rad} / \mathrm{s}]$ & Frequency $f_{\text {st }}[\mathrm{Hz}]$ \\
\hline 1 & 0.378 & 16.63 & 2.65 \\
\hline 2 & 0.344 & 18.78 & 2.99 \\
\hline
\end{tabular}

The verification of the mathematical model of the carrying structure (Fig. 6) was done by creating an FE model in the programme package SAP $2000^{\circledR}$ [17]. Using the modal analysis in SAP2000, the assumed first 12 frequencies of the carrying structure were determined. The first two frequencies are $f=(2.66 ; 3.01) \mathrm{Hz}$. By comparing the values of frequencies obtained through the mathematical model with the finite element approach and in a purely numerical way (FEM software SAP2000), coinciding well between the results of the first two frequencies and the relative deviations $\Delta=(0.38 ; 0.66) \%$ is observed. Other frequencies also coincide well. Thus, for example, for the following four frequencies the relative deviation is up to $2.5 \%$, which is very good for the spatial model.

\subsubsection{Dynamic Displacements}

The evaluation of the quality of new solutions of cranes is given based on the maximum values of dynamic displacements.

Eq. (21) for investigating the dynamic behaviour of the portal-rotating crane was solved by means of the direct, step-by-step integration method. In the Mathematica ${ }^{\circledR}$ program, the original module is written based on the Newmark integration method [18]. The time interval of integration was chosen to be $\Delta t=0.01$. Dynamic displacements for all degrees of freedom of the model were obtained, but only characteristic displacements are presented here.

The dynamic response of the portal to excitation is, before all, contained in the dynamic displacement of elastic supports of the model for different positions of the boom relative to the direction of motion of the crane. Figs. 12 through 16 present the comparative change of dynamic displacements of nodes 1 to 4 for the boom positions relative to the rectilinear motion of the portal $\varphi=0^{\circ}, 45^{\circ}, 90^{\circ}, 135^{\circ}$, and $180^{\circ}$. 


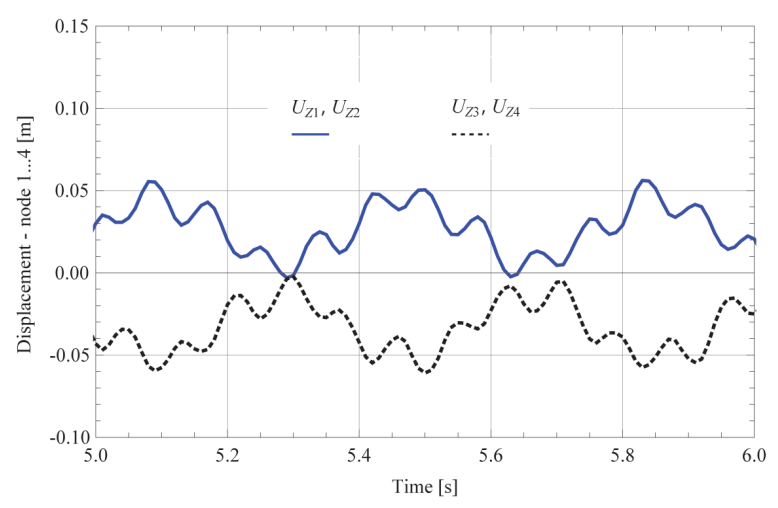

Fig. 12. Dynamic displacements of nodes 1 to $4 ; \varphi=0^{\circ}$

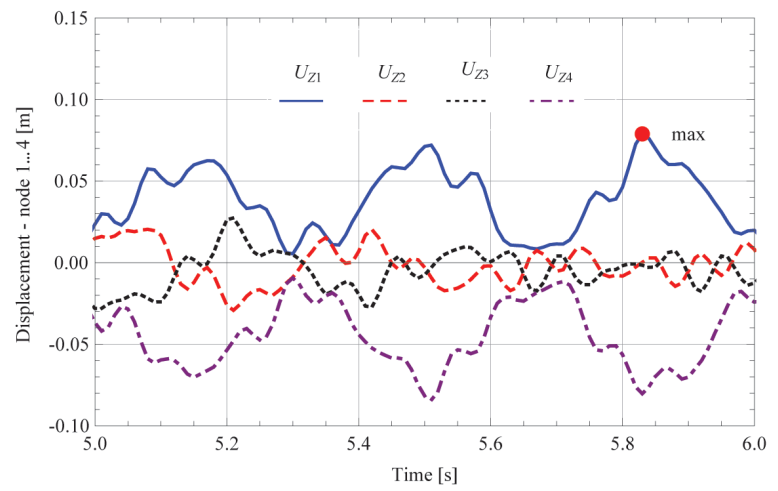

Fig. 13. Dynamic displacements of nodes 1 to $4 ; \varphi=45^{\circ}$

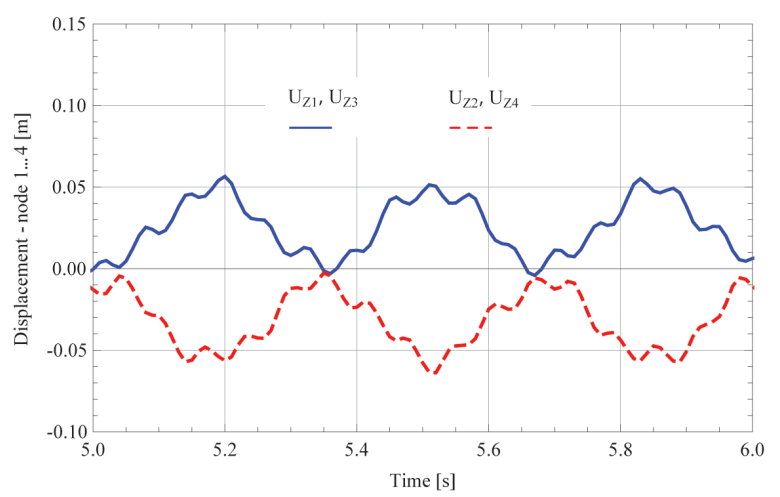

Fig. 14. Dynamic displacements of nodes 1 to $4 ; \varphi=90^{\circ}$

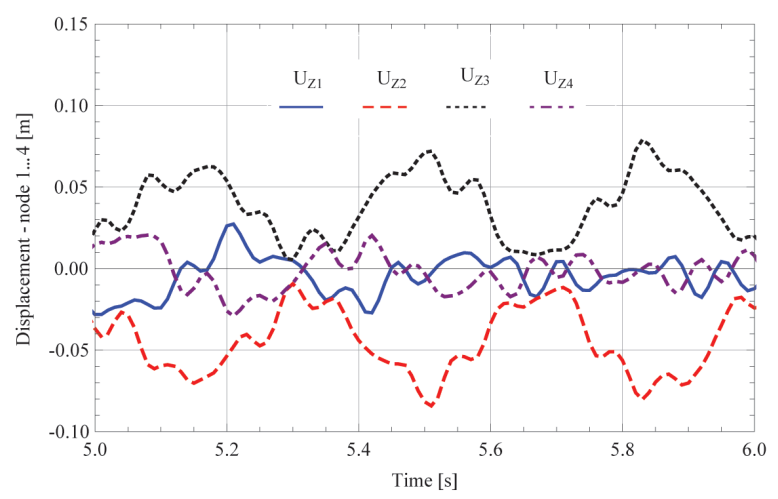

Fig. 15. Dynamic displacements of nodes 1 to $4 ; \varphi=135^{\circ}$

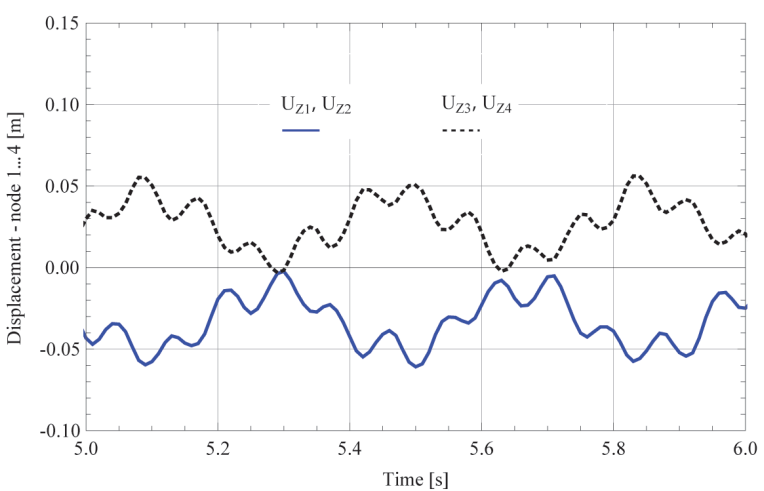

Fig. 16. Dynamic displacements of nodes 1 to $4 ; \varphi=180^{\circ}$

As the carrying structure is symmetric on one side, and as the dynamic moment is much higher in the longitudinal direction than in the lateral one, on the other side, dynamic displacements of nodes 1 and 2, i.e. 3 and 4 for the angle of the boom $0^{\circ}$ and $180^{\circ}$ almost coincide. For the angle of the boom $0^{\circ}$, displacements of nodes 1 and 2 are positive, while for the angle of the boom $180^{\circ}$ displacements of nodes 3 and 4 are positive. Analogously, displacements of nodes 1 and 3, i.e. 2 and 4 for the angle of the boom $90^{\circ}$ almost coincide. Displacements of nodes 1 and 3 are positive. For the angle of the boom $45^{\circ}$, displacement of node 1 stands out because it is positive. Analogously, for the angle $135^{\circ}$ the positive displacement of node 3 stands out.

Based on the analysis of displacements of elastic supports, Figs. 12 through 16, it was shown that the elastic support - node 1 has the largest positive displacement for the value of the angle of boom rotation of $\varphi=45^{\circ}$. The maximum displacement of node 1 is $78.93 \mathrm{~mm}$. In this case, lifting of that leg of the portal crane most often occurs in practice.

Therefore, the carrying structure of the considered portal crane is most sensitive to the boom position of $45^{\circ}$. Accordingly, this position is most relevant for describing the dynamic state of the carrying structure.

In accordance with the parameters included in the mathematical model of the considered carrying structure of the portal crane, the expected value of maximum dynamic displacement was obtained.

\section{CONCLUSIONS}

Analysis of the dynamic behaviour of portal cranes of large carrying capacities is necessary because it provides quality bases for their optimal design. The research conducted allows thorough consideration of the parameters influencing the dynamic behaviour of portal cranes. 
A combined approach, which combines equations of analytical mechanics and the finite element method, was developed. In accordance with that, dynamic spatial models of the boom and the structure of the portal crane were formed and solved. It was established that the influence of load swing is critical in portal cranes. It was shown that speed and acceleration/ deceleration of the crane influence the dynamic response of the structure. For the nominal value of the crane speed of $0.6 \mathrm{~m} / \mathrm{s}$, the obtained maximum angle of oscillation of load is $0.0815 \mathrm{rad} / \mathrm{s}$. The stability of the portal crane excited by the motion of the crane and the load swing was investigated. The dynamic response of the structure to excitation is presented through dynamic displacements of elastic supports. The boom position at which maximum displacements of elastic supports occur was established. The critical elastic support, i.e. the leg that may be lifted, was defined for this position of the boom. The maximum dynamic displacement of the critical elastic support of $78.93 \mathrm{~mm}$ was obtained.

This paper leaves some space for the continuation of the investigation of parameters influencing the dynamic behaviour of the carrying structure in portalrotating cranes and the optimization of such types of structures. Further work on the problems considered should lead to the improvement of the created models, which will include a larger number of parameters. Furthermore, this paper presents a quality basis for research into the dynamic behaviour of other types of portal rotating cranes (e.g. type $\mathrm{X}$ ).

\section{NOMENCLATURE}

$v_{c} \quad$ speeds of the moving crane $[\mathrm{m} / \mathrm{s}]$

$\ddot{x}(t)$ acceleration of the moving crane $\left[\mathrm{m} / \mathrm{s}^{2}\right]$

$\tau$ time of acceleration (deceleration) of the crane $[\mathrm{s}]$

$m_{1}$ reduced mass of the boom $[\mathrm{kg}]$

$m_{2}$ load mass $[\mathrm{kg}]$

$m_{b}$ boom mass [kg]

$g$ gravitational constant $\left[\mathrm{m} / \mathrm{s}^{2}\right]$

$L_{b}$ boom length [m]

$L_{r} \quad$ rope length [m]

$r$ distance between the boom joint and the column axis [m]

$\theta$ angle of load oscillation in the longitudinal direction [rad]

$\psi \quad$ angle of load oscillation in the lateral direction [rad]

$\varphi$ angle of column (boom) rotation $\left[{ }^{\circ}\right]$

$x \quad$ rectilinear motion of the crane $[\mathrm{m}]$

$\omega \quad$ circular frequency of the load $[\mathrm{rad} / \mathrm{s}]$

$T$ moment of rotation [Nm]

[M] structural mass matrix $[\mathrm{kg}]$
$[\mathbf{K}]$ structural stiffness matrix $[\mathrm{N} / \mathrm{m}]$

$\{\mathbf{P}(\mathbf{t})\} \quad$ external force vector $[\mathrm{N}]$

$\{\mathbf{U}\}$ displacement vector $[\mathrm{m}]$

$\{\ddot{\boldsymbol{U}}\}$ acceleration vector $\left[\mathrm{m} / \mathrm{s}^{2}\right]$

$\left[\mathbf{M}_{\mathbf{u u}}\right]$ mass submatrix of the structure in the direction of unknown displacements $[\mathrm{kg}]$

$\left[\mathbf{K}_{\mathbf{u u}}\right]$ stiffness submatrix of the structure in the direction of unknown displacements [kg]

[k] stiffness matrix of elastic supports [N/m]

$k \quad$ stiffness of elastic supports [N/m]

$\left\{\mathbf{P}_{\mathbf{u}}(\mathbf{t})\right\}$ external subvector of forces in the direction of unknown displacements [N]

$\left\{\mathbf{U}_{\mathbf{u}}\right\}$ displacement vector in the direction of unknown displacements [m]

$\left\{\ddot{\boldsymbol{U}}_{\mathbf{u}}\right\} \quad$ acceleration vector in the direction of unknown displacements $\left[\mathrm{m} / \mathrm{s}^{2}\right]$

$L \quad$ length of the portal base [m]

$B \quad$ width of the portal base [m]

$H$ height of the leg [m]

$H_{0}$ distance between the upper and the lower supports of the boom [m]

$l_{n} \quad$ length of the finite element [m]

$A_{n}$ cross-sectional area[m²]

$I_{x n} \quad$ moment of inertia of cross section the $x$ axis $\left[\mathrm{m}^{4}\right]$

$I_{y n} \quad$ moment of inertia of cross-section the $y$ axis $\left[\mathrm{m}^{4}\right]$

$I_{z n} \quad$ moment of inertia of cross-section the $z$ axis $\left[\mathrm{m}^{4}\right]$

$\rho$ mass density of material $\left[\mathrm{kg} / \mathrm{m}^{3}\right]$

$E \quad$ Young's modulus $\left[\mathrm{N} / \mathrm{m}^{2}\right]$

$G$ slipping module $\left[\mathrm{N} / \mathrm{m}^{2}\right]$

$\omega_{s t}$ circular frequency of structure $[\mathrm{rad} / \mathrm{s}]$

$f_{s t} \quad$ frequency of structure $[\mathrm{Hz}]$

$\Delta t \quad$ time interval of integration [s]

$p \quad$ total number of time steps [-]

\section{REFERENCES}

[1] Jerman, B., Hribar, A. (2013). Dynamics of the mathematical pendulum suspended from a moving mass. Tehnički vjesnik Technical Gazette, vol. 20, no. 1, p. 59-64.

[2] Fryba, L. (1999). Vibration of Solids and Structures under Moving Loads. 3rd ed., Thomas Telford, London, D0I:10.1680/ vosasuml.35393.

[3] Gašić, V., Zrnić, N., Rakin, M. (2012). Consideration of a Moving Mass Effect on Dynamic Behaviour of a Jib Crane Structure. Tehnički vjesnik - Technical Gazette, vol. 19, no. 1, p. 115-121.

[4] Raubar, E., Vrančić, D. (2012). Anti-Sway System for Shipto-Shore Cranes. Strojniški vestnik - Journal of Mechanical Engineering, vol. 58, no. 5, p. 338-344, Dol:10.5545/svjme.2010.127.

[5] F.E.M 1.001. (1998). Rules for the Design of Hoisting Appliances. European Materials Handling Federation, Brussels.

[6] Osiński, M., Wojciech, S. (1998). Application of Nonlinear Optimisation Methods to effect Input Shaping of the Hoist 
Drive of an Off-Shore Crane. Nonlinear Dynamics, vol. 17, no. 4, p. 369-388, D0I:10.1023/A:1008333417693.

[7] Jovanović, M., Radoičić, G., Milić, P. (2009). Selection of Finite Elements Considering Loadcases and Geometry. Proceedings of the XIXth International Conference MHCL, p. 61-66.

[8] Sun, Y., Li, D. (2012). Dynamic analysis and design method study on the combined-boom system of portal crane. Applied Mechanics and Materials, vol. 152-154, p. 1645-1649, Dol:10.4028/www.scientific.net/AMM.152-154.1645.

[9] Marinović, I., Sprečić, D., Jerman, B. (2012). A slewing crane payload dynamics. Tehnički vjesnik - Technical Gazette, vol. 19, no. 4, p. 907-916.

[10] Maczyński, A., Szczotka, M. (2002). Comparison of Models for Dynamic Analysis of a Mobile Telescopic Crane. Journal of Theoretical and Applied Mechanics, vol. 40, no. 4, p. 10511074.

[11] Fujioka, D., Rauch, A., Singhose, W. (2009). Selection of finite elements considering loadcases and geometry doublependulum payloads. American Control Conference, p. 31363014.

[12] Gustafsson, T. (1996). On the design and implementation of a rotary crane controller. European Journal of Control, vol. 2, no. 3, p. 166-175, Dol:10.1016/S0947-3580(96)70042-0.

[13] Huimin, O., Naoki, U., Shigenori, S. (2010). Anti-Sway control of rotary crane only by horizontal boom motion. IEEE International Conference on Control Applications, p. 591-595.

[14] Vasiljević, R.; Savković, M.; Bulatović, R. (2013). The Approaches to the Mathematical-mechanical Modeling Supporting Construction. IMK-14 - Research \& Development in Heavy Machinery, vol. 19, no. 1, p. EN29-38.

[15] Gašic, V. (2004). Dynamic behaviour identification of bridge type stacker - reclaimer with bucket chain booms in power plants. M.Sc. thesis, Faculty of Mechanical Engineering, Belgrade.

[16] Vasiljević, R., Gašić, M. (2015). The Dynamic Model of the Boom Portal Cranes. IMK-14 - Research \& Development in Heavy Machinery, vol. 21, no. 4, p. EN125-130.

[17] Computers and Structures Inc. (2009). SAP2000 ${ }^{\circledR}$ Analysis reference manual, Berkeley.

[18] Bathe, K.J. (1996). Finite Element Procedures. Prentice-Hall, New Jersey.

\section{APPENDIX}

\subsection{Equations (2a) and (2b)}

\subsubsection{Equations (2a)}

The differential equation of relative motion of the mass $m_{2}$ in the longitudinal direction reads:

$$
m_{2} \vec{a}_{r}=\vec{F}_{p x}^{i n}+\vec{F}_{p z}^{i n}+\vec{G}_{2}+\vec{S} .
$$

Intensity of inertial forces:

$$
\begin{gathered}
\vec{F}_{p x}^{i n}=m_{2} \ddot{x}(t) \cos \varphi, \\
\vec{F}_{p z}^{i n}=m_{2} \ddot{z}(t) .
\end{gathered}
$$

Eq. (A1) is projected onto the axes $t$ and $n$ of the natural trihedron:

$$
\begin{aligned}
m_{2} L_{r} \ddot{\theta}(t) & =-G_{2} \sin \theta(t)-m_{2} \ddot{x}(t) \cos \varphi \cos \theta(t)+ \\
& +m_{2} \ddot{z}(t) \sin \theta(t), \\
m_{2} L_{r} \ddot{\theta}(t) & =-G_{2} \cos \theta(t)-m_{2} \ddot{x}(t) \cos \varphi \sin \theta(t)+ \\
& +m_{2} \ddot{z}(t) \cos \theta(t) .
\end{aligned}
$$

For $\ddot{z}=0$ Eqs. (A4) and (A5) obtain the form:

$$
\begin{aligned}
& m_{2} L_{r} \ddot{\theta}(t)=-G_{2} \sin \theta(t)-m_{2} \ddot{x}(t) \cos \varphi \cos \theta(t), \\
& m_{2} L_{r} \ddot{\theta}(t)=-G_{2} \cos \theta(t)-m_{2} \ddot{x}(t) \cos \varphi \sin \theta(t) .
\end{aligned}
$$

From Eq. (A6) is determined by the law of oscillation of cargo in the longitudinal direction:

$$
\ddot{\theta}(t)+\frac{g}{L_{r}} \sin \theta(t)=-\frac{1}{L_{r}} \ddot{x}(t) \cos \varphi \cos \theta(t) .
$$

The approximation $\sin \theta \approx \theta$ and $\cos \theta \approx 1$ is introduced:

$$
\ddot{\theta}(t)+\frac{g}{L_{r}} \theta(t)=-\frac{1}{L_{r}} \ddot{x}(t) \cos \varphi,
$$

where $g / L_{r}$ represents the quadrant circular frequency of the $\operatorname{load} \omega^{2}$.

The replacement $a=\ddot{x}(t)$ is introduced:

$$
\ddot{\theta}(t)+\omega^{2} \theta(t)=-\frac{1}{L_{r}} a(t) \cos \varphi .
$$

Laplace transformation:

$$
\begin{gathered}
L\{\theta(s)\}\left(s^{2}+\omega^{2}\right)=-\frac{1}{L_{r}} L\{a(s)\} \cos \varphi, \\
L\{(s)\}=-\frac{1}{L_{r}} L\left\{\frac{a(s)}{\left(s^{2}+\omega^{2}\right)}\right\} \cos \varphi .
\end{gathered}
$$

The expression for acceleration in the Laplace domain can be represented in the following form:

$$
a(s)=\frac{h}{s}\left(1-e^{t_{2} s}-e^{t_{3} s}+e^{t_{4} s}\right) .
$$

Eq. (A12) obtains the form:

$$
L\{\theta(s)\}=-\frac{h}{L_{r}} L\left\{\frac{1-e^{t_{2} s}-e^{t_{3} s}+e^{t_{4} s}}{s\left(s^{2}+\omega^{2}\right)}\right\} \cos \varphi .
$$

Finally, the law of load oscillation in the longitudinal direction is obtained by transforming the Eq. (A14) in the time domain by using the inverse Laplace transform (Mathematica ${ }^{\circledR}$ program used):

$$
\begin{aligned}
\theta(t) & =-\frac{h}{L_{r} \omega^{2}} \cos \varphi\{(1-\cos \omega t)- \\
& -\left[1-\cos \omega\left(t-t_{2}\right)\right] \text { HeavisideTheta }\left(t-t_{2}\right)- \\
& -\left[1-\cos \omega\left(t-t_{3}\right)\right] \text { HeavisideTheta }\left(t-t_{3}\right)+ \\
& \left.+\left[1-\cos \omega\left(t-t_{4}\right)\right] \text { HeavisideTheta }\left(t-t_{4}\right)\right\} .
\end{aligned}
$$




\subsubsection{Equations (2b)}

The differential equation of relative motion of the mass $m_{2}$ in the side (lateral) direction reads:

$$
m_{2} \vec{a}_{r}=\vec{F}_{p y}^{i n}+\vec{F}_{p z}^{i n}+\vec{G}_{2}+\vec{S} .
$$

Intensity of inertial forces:

$$
\begin{gathered}
\vec{F}_{p y}^{i n}=m_{2} \ddot{x}(t) \sin \varphi, \\
\vec{F}_{p z}^{i n}=m_{2} \ddot{z}(t) .
\end{gathered}
$$

Further, the Eq. (A16) solved by analogue Eq. (A1). The law of load oscillation in the side direction reads:

$$
\begin{aligned}
\psi(t) & =-\frac{h}{L_{u} \omega^{2}} \sin \varphi\{(1-\cos \omega t)- \\
& -\left[1-\cos \omega\left(t-t_{2}\right)\right] \text { HeavisideTheta }\left(t-t_{2}\right)- \\
& -\left[1-\cos \omega\left(t-t_{3}\right)\right] \text { HeavisideTheta }\left(t-t_{3}\right)+ \\
& \left.+\left[1-\cos \omega\left(t-t_{4}\right)\right] \text { HeavisideTheta }\left(t-t_{4}\right)\right\} .
\end{aligned}
$$

\subsection{Equations (24)}

Eq. (24) reads:

$$
\mathbf{K}_{\mathrm{uu}}=\left[\mathbf{K}_{\mathbf{u u}}\right]_{82 \times 82}+[\mathbf{k}]_{16 \times 16} .
$$

In order to execute Eq. (24), the stiffness matrix of elastic supports $[\mathbf{k}]_{16 \times 16}$ is, by adding zero rows and zero columns, extended to the matrix with dimensions $82 \times 82$, except for 16 degrees of freedom of four nodes:

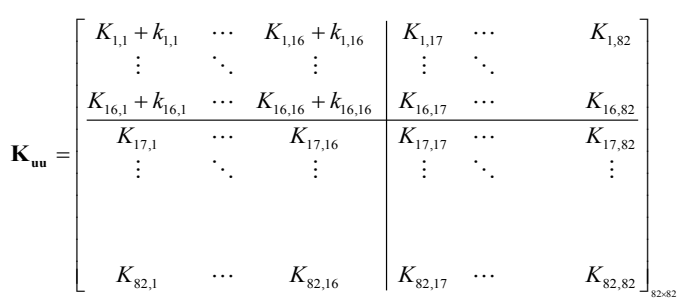

\subsection{FE model in SAP2000 ${ }^{\circledR}$ software}

The FE model of the structure of the portal crane is shown in Fig. A1. Model is created in FE package SAP2000 ${ }^{\circledR}$. The first 2 mode shapes of the structure are shown in Figs. A1 and A2.

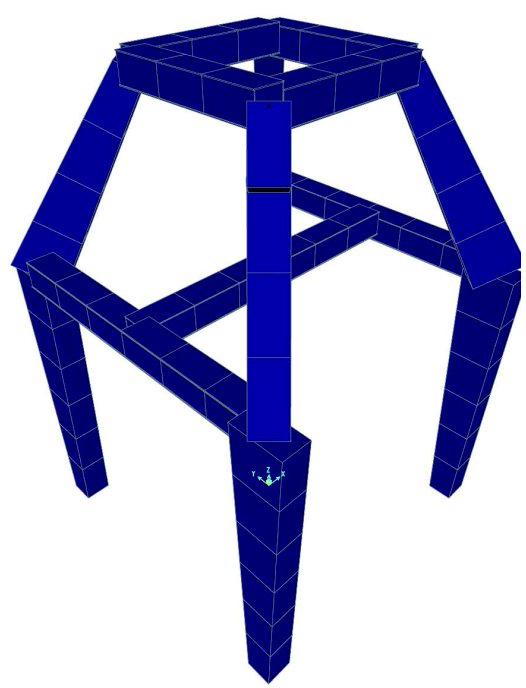

Fig. A1. FE model of the structure of type " $H^{\prime \prime}$

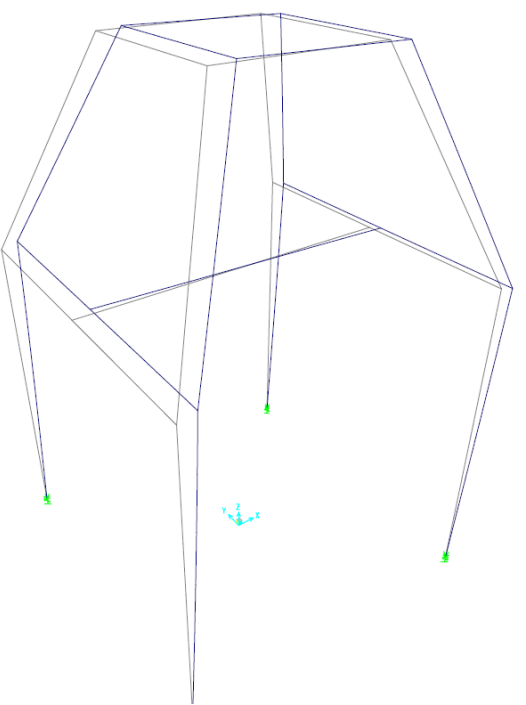

Fig. A2. $1^{\text {st }}$ mode, $f_{1}=2.66 \mathrm{~Hz}$

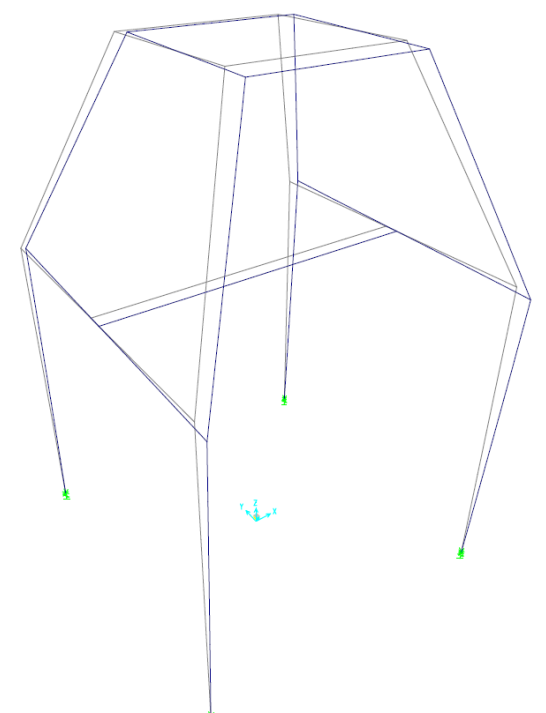

Fig. A3. $2^{\text {nd }}$ mode, $f_{2}=3.01 \mathrm{~Hz}$ 